\title{
Nanoscale Molecular Reorganization of the Inhibitory Postsynaptic Density Is a Determinant of GABAergic Synaptic Potentiation
}

\author{
Francesca Pennacchietti, ${ }^{1,4,6 *}$ Sebastiano Vascon, ${ }^{2 *}$ (Thierry Nieus, ${ }^{3 *}$ (C) Christian Rosillo, ${ }^{3}$ Sabyasachi Das, ${ }^{3}$ \\ @-Shiva K. Tyagarajan, ${ }^{5}$ Alberto Diaspro, ${ }^{1,4,6}$ @Alessio Del Bue, ${ }^{2}$ ¿Enrica Maria Petrini, ${ }^{3}$ @Andrea Barberis, ${ }^{3} \dagger$ \\ and Francesca Cella Zanacchi ${ }^{1,4} \dagger$ \\ ${ }^{1}$ Nanoscopy Department, ${ }^{2}$ Pattern Analysis and Computer Vision Department, ${ }^{3}$ Neuroscience and Brain Technologies Department, and ${ }^{4}$ Nikon Imaging \\ Center, Fondazione Istituto Italiano di Tecnologia, 16163 Genoa, Italy, Institute of Pharmacology and Toxicology, University of Zurich, 8057 Zurich, \\ Switzerland, and ${ }^{6}$ Università di Genova, Department of Physics, 16146 Genoa, Italy
}

Gephyrin is a key scaffold protein mediating the anchoring of GABAA receptors at inhibitory synapses. Here, we exploited superresolution techniques combined with proximity-based clustering analysis and model simulations to investigate the single-molecule gephyrin reorganization during plasticity of inhibitory synapses in mouse hippocampal cultured neurons. This approach revealed that, during the expression of inhibitory LTP, the increase of gephyrin density at postsynaptic sites is associated with the promoted formation of gephyrin nanodomains. We demonstrate that the gephyrin rearrangement in nanodomains stabilizes the amplitude of postsynaptic currents, indicating that, in addition to the number of synaptic GABAA receptors, the nanoscale distribution of GABAA receptors in the postsynaptic area is a crucial determinant for the expression of inhibitory synaptic plasticity. In addition, the methodology implemented here clears the way to the application of the graph-based theory to single-molecule data for the description and quantification of the spatial organization of the synapse at the single-molecule level.

Key words: clustering; GABAA receptors; gephyrin; model simulations; PALM; plasticity; STORM

Significance Statement

The mechanisms of inhibitory synaptic plasticity are poorly understood, mainly because the size of the synapse is below the diffraction limit, thus reducing the effectiveness of conventional optical and imaging techniques. Here, we exploited superresolution approaches combined with clustering analysis to study at unprecedented resolution the distribution of the inhibitory scaffold protein gephyrin in response to protocols inducing LTP of inhibitory synaptic responses (iLTP). We found that, during the expression of iLTP, the increase of synaptic gephyrin is associated with the fragmentation of gephyrin in subsynaptic nanodomains. We demonstrate that such synaptic gephyrin nanodomains stabilize the amplitude of inhibitory postsynaptic responses, thus identifying the nanoscale gephyrin rearrangement as a key determinant for inhibitory synaptic plasticity.

\section{Introduction}

In the last few years, single-molecule localization (SML) techniques have been applied to address several biological questions requiring the observation of subcellular structures beyond the

Received Feb. 16, 2016; revised Dec. 22, 2016; accepted Dec. 31, 2016.

Author contributions: A.D., A.D.B., E.M.P., A.B., and F.C.Z. designed research; F.P., S.V., T.N., C.R., S.D., F.C.Z., and E.M.P. performed research; S.K.T. contributed unpublished reagents/analytic tools; F.P., S.V., T.N., A.D.B., E.M.P., and F.C.Z. analyzed data; A.d.B., A.B., and F.C.Z. wrote the paper.

This work was supported by Telethon-Italy (Grant GGP11043 to A.B.), Compagnia di San Paolo (Grant ROL-4318 to A.B.), and Compagnia di San Paolo (Grant ROL-9687 to Marti Duocastella and A.B.).

The authors declare no competing financial interests.

*F.P., S.V., and T.N. contributed equally to this work.

†A.B. and F.C.Z. contributed equally to this work. diffraction limit (Szymborska et al., 2013, Xu et al., 2013). By providing imaging capability with resolution never attempted before (10-30 nm), SML techniques (Deschout et al., 2014a) represent a powerful tool with which to quantify the number and the distribution of molecules in several biological systems (Deschout et al., 2014b). As a general rule, quantitative singlemolecule analysis basically relies on two main aspects: a proper fluorescent tag enabling a reliable detection and a statistical tool

Correspondence should be addressed to either Andrea Barberis, Neuroscience and Brain Technologies Department, or Francesca Cella Zanacchi, Nanoscopy Department, Fondazione Istituto Italiano di Tecnologia, Via Morego 30, 16163 Genoa, Italy. E-mail: andrea.barberis@iit.it or francesca.cella@iit.it.

DOI:10.1523/JNEUROSCI.0514-16.2016

Copyright $\odot 2017$ the authors $\quad 0270-6474 / 17 / 371747-10 \$ 15.00 / 0$ 
able to identify the spatial features of interest. In this context, among the great variety of SML techniques, PALM (photoactivatable localization microscopy) imaging represents an ideal approach to "count" proteins because the use of photoactivatable fluorescence proteins (PA-FPs) provides high labeling specificity and a 1:1 ratio with the protein of interest (Fernández-Suárez and Ting, 2008). Furthermore, an irreversible PA-FP such as mEos allows the direct correlation between the photoactivation process and the detection of the protein of interest, leading to robust quantitative measurements. Imaging at the nanoscale level implies new challenges for the development of custom and highly efficient computational tools to quantify aggregations of proteins by solving clustering problems involving a high number of molecules with arbitrary densities. In neuronal cells, quantitative SML has been applied recently to assess the number and the spatial distribution of synaptic proteins (Dani et al., 2010, Sigrist and Sabatini, 2012, Nair et al., 2013, Specht et al., 2013) and to reveal the subunit composition of neurotransmitter synaptic receptors (Ulbrich and Isacoff, 2007, Durisic et al., 2012, MacGillavry et al., 2013). At inhibitory synapses, quantitative SML techniques have been exploited to clarify the stoichiometry between the anchoring protein gephyrin and synaptic GABAA receptors (Specht et al., 2013), thus allowing a new insight of the molecular organization of the inhibitory postsynaptic density. Although some aspects of postsynaptic protein assembly at inhibitory synapses are beginning to be unraveled, the molecular rearrangements responsible for the activity-dependent scaling of inhibitory synaptic strength are still poorly understood, mainly due to the lack of appropriate quantitative imaging and computational tools. Recently, it has been reported that a form of chemical long term potentiation (LTP) of inhibitory synapses relies on the promoted accumulation of gephyrin at synaptic areas, thereby enhancing the clustering of synaptic GABAA receptors and potentiating unitary GABAergic synaptic currents (Petrini et al., 2014). By combining superresolution approaches with a novel clustering analysis, the present study reveals at unprecedented resolution the inner organization of the inhibitory postsynaptic scaffold, providing for the first time the molecular quantification of the rearrangements of synaptic gephyrin molecules during inhibitory LTP (iLTP). We show here that iLTP enhances the formation of gephyrin nanodomains within the synaptic area. Using a model simulations approach, we demonstrate that such gephyrin nanoscale compartmentalization reduces the variability of synaptic responses. These findings reveal that the nanoscale redistribution of the scaffold protein gephyrin is a key event in the expression of inhibitory synaptic potentiation.

\section{Materials and Methods}

\section{Biological protocols}

The same preparation, imaging and analysis protocols were applied to all samples.

Plasmid constructs. mEOS2-Gephyrin was made by PCR amplifying mEOS2 sequence from Addgene plasmid \#20341, pRSETa mEos2 using PCR primers to introduce restriction sites Nhel and XhoI. eGFP was excised out of eGFPC2-GephyrinP1 using NheI and XhoI and replaced with a PCR-amplified mEOS2 sequence. Gephyrin-mEos3.2 was obtained by site-directed mutagenesis of the gephyrin-mEos 2 plasmid. The mutations I102N, H158E, and Y189A were introduced with the Agilent Technologies mutagenesis kit. The plasmid was sequence confirmed.

Primary neuronal cultures and transfection. Cultures of hippocampal neurons were prepared from C57BL/6J mice of either sex as described previously (Petrini et al., 2014). Neurons were transfected at $7 \mathrm{~d}$ in vitro (DIV) using Effectene (Qiagen) following the manufacturer's protocol. Experiments were performed at 16-18 DIV.
iLTP induction and drug treatments. NMDA receptor (NMDAR) activation was used to induce iLTP chemically, as described previously (Petrini et al., 2014). Briefly, neurons were incubated in a recording solution containing the following (in mM): $145 \mathrm{NaCl}, 2 \mathrm{KCl}, 2 \mathrm{CaCl}_{2}, 2 \mathrm{MgCl}_{2}, 10$ glucose, and 10 HEPES, pH 7.4, supplemented with $20 \mu \mathrm{M}$ NMDA (Sigma-Aldrich) and $10 \mu \mathrm{M}$ CNQX (Tocris Bioscience) for $2 \mathrm{~min}$ and then allowed $18 \mathrm{~min}$ recovery in the recording solution. In control experiments, NMDA and CNQX were omitted. To prevent iLTP, neurons pretreated with the NMDA antagonist APV (50 $\mu$ m; Tocris Bioscience) for $10 \mathrm{~min}$ were incubated with the stimulating solution (NMDA and CNQX) supplemented with $50 \mu \mathrm{M}$ APV and compared with stimulated samples without APV and with matched controls.

Sample preparation. To localize presynaptic terminals by fluorescence, live immunolabeling of vGAT was performed before the stimulation by incubating neurons for $20 \mathrm{~min}$ in the culture medium with an anti-vGAT antibody coupled to Oyster650 directed against the luminal domain of vGAT (Synaptic Systems). Alternatively, for stochastic optical reconstruction microscopy (STORM) experiments, presynaptic terminals were immunoprobed with the anti-vGAT antibody along with the mAb7a antibody (both from Synaptic Systems) to localize endogenous gephyrin or with the anti-GABAA receptor $\alpha 1$ subunit (Alomone), followed by specie-specific secondary antibodies coupled to Atto520 and Alexa Fluor 647 (both from Thermo Fisher Scientific), respectively. After the recovery period, neurons were fixed in $4 \%$ PFA for $13 \mathrm{~min}$.

\section{Electrophysiology}

Spontaneous inhibitory postsynaptic currents (sIPSCs) were recorded in the whole-cell configuration of the patch-clamp technique. External recording solution contained the following (in mM): $145 \mathrm{NaCl}, 2 \mathrm{KCl}, 2$ $\mathrm{CaCl}_{2}, 2 \mathrm{MgCl}_{2}, 10$ glucose, and 10 HEPES, $\mathrm{pH}$ 7.4. Patch pipettes, pulled from borosilicate glass capillaries (Hilgenberg), had a 4-5 MO resistance when filled with intracellular recording solution containing the following (in mM): $150 \mathrm{KCl}, 1 \mathrm{CaCl}_{2}, 2 \mathrm{MgCl}_{2}, 1$ EGTA, 10 HEPES, and $2 \mathrm{Na}_{2} \mathrm{ATP}$ at $300 \mathrm{mOsm}$ and $\mathrm{pH} 7.2$ with $\mathrm{KOH}$. Currents were acquired using Clampex 10.0 software (Molecular Devices). sIPSCs were recorded at room temperature from a holding potential of $-60 \mathrm{mV}$ in the presence of CNQX (10 mM) to isolate GABAergic events. sIPSCs were detected by using the scaled sliding template detection algorithm implemented in pClamp 10 by setting the detection criterion value to 5 . The coefficient of variation $(\mathrm{CV})$ was computed at the current peak of sIPSCs recorded before and $18 \mathrm{~min}$ after the NMDA application.

\section{Single-molecule superresolution imaging}

SML imaging. Single-molecule imaging was performed with a superresolution microscope Nikon N-STORM equipped with a $100 \times$ oilimmersion objective (numerical aperture 1.40) and an Andor iXon DU-897E-CS0 BV EMCCD camera running at $20 \mathrm{~Hz}$. Imaging was performed in PBS. In each acquisition run, 20,000 frames of a $20 \times 20 \mu \mathrm{m}$ were recorded while maintaining the $z$ position with the Nikon perfect focus system. The duration of the acquisition was the same in all experiments. The intensity of the activation $405 \mathrm{~nm}$ laser (Coherent CUBE $405-100 \mathrm{~mW}$ ) was $<0.25 \mathrm{~W} / \mathrm{cm}^{2}$ to ensure the single-molecule regime and to avoid misleading localizations; the $561 \mathrm{~nm}$ excitation laser (Coherent Sapphire OPSL $561 \mathrm{~nm}$ ) intensity was $\sim 0.3 \mathrm{~kW} / \mathrm{cm}^{2}$; the $647 \mathrm{~nm}$ excitation laser intensity was $\sim 0.8 \mathrm{~kW} / \mathrm{cm}^{2}$. An emission filter ET605/70 (Chroma) was added after the four-color dichroic mirrors (ZET405/488/ 561/647; Chroma) and a multiband-pass filter (ZT405/488/561/647; Chroma) was used to filter the fluorescence emission. SML fitting was conducted with the Nikon-integrated routine taking into account drift and chromatic aberrations. In the superresolution image reconstruction, each molecule is represented by a Gaussian spot with the SD obtained from the single-molecule fit and by an amplitude value related to the photon number. To compensate for fluorophore blinking, we followed an approach described previously (Annibale et al., 2011) by estimating the empirical dark-state lifetime calculated as the fit of the normalized experimental number of localized molecules plotted as a function of the dark time td, where the spatial interval to consider a single-molecule reappearance was set to $15 \mathrm{~nm}$ radius, a value corresponding to half of to the mean localization precision. Our estimation of the dark-state lifetime 
of mEos3.2 ( $\left.\tau_{\text {off }} \sim 0.13 \pm 0.01 \mathrm{~s}\right)$ was consistent with the values reported for the other variant of the PA-FP family.

\section{Clustering algorithm}

In the dominant set (DS) approach, a set of points (molecule localizations) is considered as a graph in which the vertices are the points and the edges connecting pairs of nodes are weighted by the similarity between them (closeness). This is formalized (Pavan and Pelillo, 2007) as an undirected, edge-weighted graph according to the following:

$$
G=(V, E, \omega)
$$

with no self-loop, in which $V$ is the set of nodes, $E$ is the set of edges, and $\omega$ the similarity function quantifying the closeness of pairs of nodes as follows:

$$
\omega_{i, j}=e^{-\frac{d(i, j)}{2 \sigma^{2}}}
$$

where $d(i, j)$ is a distance function, the Euclidean distance between two molecules of localization $d(i, j)=i-j$ and $\sigma$ acts as a scaling factor. Each cluster is given by the optimization of a quadratic function in $x$ (Standard Quadratic Assignment Program) as follows:

$$
\pi(x)=x^{T} A x
$$

where each element of the similarity matrix $A$ corresponds to $\omega_{i, j}$. Maximizing the function $(x)$, by finding $\bar{x}=\arg \max \pi(x)$, means to find clusters that have a good assignment between nodes and thus a high similarity. This is represented by the cohesiveness value $=\bar{x}^{T} A \bar{x}$. The algorithm searches for a partition into the graph that maximizes the cohesiveness (the goodness of a cluster). In this way, the parameter $\theta$ acts as a threshold and only clusters with high cohesiveness (and thus higher clustering goodness) are validated and distinguished from noise. Once the set of valid clusters are found, to avoid oversegmentation, we introduced a "merging" step that fuses the clusters fulfilling the criteria as follows:

(1) Their centroid $\mu_{\mathrm{i}}$ and $\mu_{\mathrm{h}}$ of two clusters, $i$ and $h$, are close enough: $\mu_{i}-\mu_{h} \leq d_{\text {merge; }}$ and

(2) the ratio $\tau(i, h)=\frac{\varphi_{i} \cap \varphi_{h}}{\varphi_{i} \cup \varphi_{h}}$ is greater or equal to $\%_{\text {merge }}$. where $\mu$ is the centroid of the $i$ th cluster $\left(\mu_{i}=\frac{1}{k_{i}} \sum_{j=1}^{k_{i}} m_{j}\right)$ and the convex hull $\psi_{\mathrm{i}}$ is the smallest convex set that contains the molecules of the $i$ th cluster (therefore, $\psi_{\mathrm{i}}$ contains the set of corner points of the hull). The value $\tau(i, h)$ represents the ratio of the intersection to the union of the areas of the two convex hulls. Its range is $[0,1]$ where 0 means no overlap and 1 corresponds to complete overlap. Subsequently, to take into account the variability of gephyrin clusters while avoiding overassociation, further filters were added in the algorithm. A dispersion filter, to remove the clusters having a variance greater than the mean variance of the clusters and a size filter to discard the clusters composed by a fewer number of points. Furthermore, a filter was applied for the estimation of the cluster area and the localizations exhibiting $<2$ nearest neighbors within a radius equal to the localization precision $(\sim 30 \mathrm{~nm})$ were excluded. A blinded analysis was performed for the iLTP experiments.

\section{Computer modeling of synaptic responses}

The concentration of the GABA neurotransmitter following the release process was described by the deterministic equation as described previously (Petrini et al., 2011)

$$
[G A B A](r, t)=\frac{M}{\alpha\left(4 \pi D^{\prime} t\right)^{1.5}} e^{-r^{2} /\left(4 D^{\prime} t\right)}
$$

where $M$ is the number of neurotransmitter molecules released, $\alpha$ the volume fraction, $r$ the distance from the release site, $D^{\prime}$ the diffusion coefficient corrected by the tortuosity (i.e., $D^{\prime}=D / \lambda^{2}$ ), and $t$ the elapsed time from the synaptic release, at $t=0$. $M$ was set to 500 molecules to mimic mild neurotransmitter release. Several sets of model simulations were repeated, varying alternatively the number of molecules $(500,2000$, and 6000 molecules $)$ and the radius of the synaptic disk $(0.2$ and $0.3 \mu \mathrm{m})$.
For each trial, the position of the release site was changed randomly on the synaptic disk with a uniform distribution (100 repetitions). The Jones and Westbrook model (Jones and Westbrook, 1995) was adopted to simulate the kinetics of GABAergic synaptic currents. This kinetic scheme includes 7 states: closed (R), singly bound-closed (AR), doubly bound-closed $\left(A_{2} R\right)$, singly bound-open $\left(A^{*}\right)$, doubly bound-open $\left(A_{2} R^{*}\right)$, singly bound-desensitized $(A D)$, and doubly bound-desensitized $\left(\mathrm{A}_{2} \mathrm{D}\right)$. The transition rate constants among states have been optimized to match the amplitude and time course of mean GABAergic IPSCs recorded in mature hippocampal cultured neurons (Petrini et al., 2011). The occupancy probability of each state of the receptors was calculated by numerically solving a set of differential equations associated with the aforementioned model with an integration time step of $0.01 \mathrm{~ms}$. For each repetition, the peak of the open state (sum of the singly and doubly bound open states) and the CV (i.e., SD divided by the mean of the peak open state) were computed. Considering each receptor with a radius of $10 \mathrm{~nm}$, two fundamental receptor distributions were studied: (1) Gaussian distribution (monospot synapse); in which the density of the receptors decreases exponentially from the center to the edge of the synaptic disk (see Fig. 4A, gray), and (2) nanodomain receptor distribution (multispot synapse), in which receptors were confined into eight or four circular nanodomains distributed randomly over the synaptic disk (see Fig. $4 A$, blue and green, respectively). An additional configuration in which 4 nanodomains are fixed with equal distance $(0.1 \mu \mathrm{m})$ from the center of the synaptic disk was tested (see Fig. $4 A$, red). Nanodomains were set with a radius of $40 \mathrm{~nm}$. Each synapse was populated with an equal number of receptors ( 40 receptors). Therefore, the multispot configurations with four and eight nanodomains contained 10 and five receptors, respectively. The same spatial configurations were also studied in conditions of $20 \%$ receptor increase (i.e., 48 receptors) to match the conditions observed during iLTP (Petrini et al., 2014). In the multispot configurations, the 48 receptors were evenly distributed among the nanodomains (i.e., 12 receptors in four nanodomains and six receptors in eight nanodomains). For each synapse configuration shown in Figure 4A, 50 different rearrangements of the receptors were analyzed. In the nanodomain random configurations, each rearrangement allowed variation of both the receptor distribution within the nanodomains and the position of the nanodomains in the synaptic disk. In these configurations, to sample the synaptic disk space properly, we avoided any intersection among the nanodomains. In addition, we prevented the nanodomains from crossing the synaptic disk borders. In this scenario, the nanodomains could span any configuration, ranging from well separated to abutting. The overlap among the receptors was prevented in all configurations by introducing a slight repulsive bouncing among the receptors before starting the simulations. Receptor clustering in simulated nanodomains was defined using the DBSCAN algorithm. In the particular cases of adjacent receptor nanodomains, receptor clustering was optimized to obtain the best cluster separation based on the silhouette coefficient $(S)$, a parameter that estimates the degree of receptor clustering and ranges between -1 (poor clustering) and 1 (optimal clustering). Simulated nanodomains were also clustered using the KMeans algorithm. This "basic" approach yielded similar results to the DBSCAN algorithm (the Pearson correlation coefficient of the silhouette was 0.62 0.99 ), indicating that our results were algorithm independent. In the fixed four nanodomains configuration, the 50 realizations varied only receptor localization within each fixed nanodomain. The variable arrangements of the receptors allowed relating the different degrees of clustering of the receptors to the variability of the current peak responses.

\section{Statistics}

Values are given as means \pm SD. Normally distributed datasets were compared using the unpaired two-tailed Student's $t$ test and the difference between the empirical distribution functions were evaluated with the Kolmogorov-Smirnov test. Comparison of more than two nonparametric datasets was performed with one-way ANOVA (Kruskal-Wallis test followed by Dunns' post test). Indications of significance correspond to $p$-values $<0.05\left(^{*}\right), p<0.01\left(^{* *}\right), p<0.001\left(^{* * *}\right)$, and nonsignificant (ns). For observation of the nanoscale inner organization of gephyrin, 
A
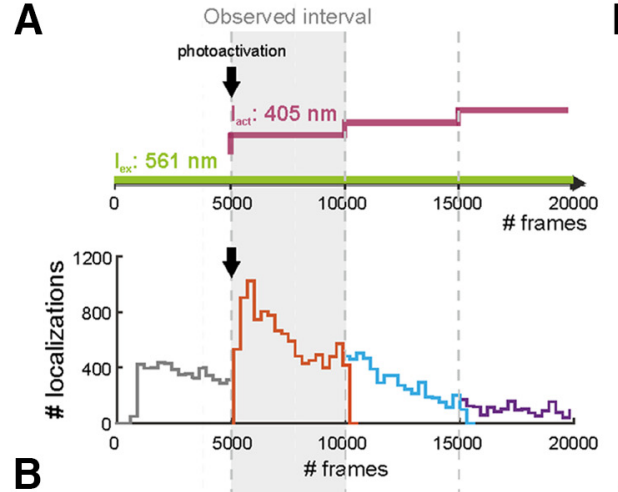

$B$

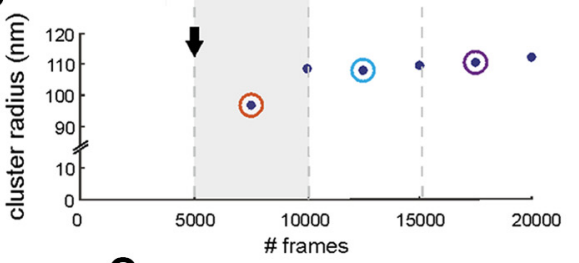

C
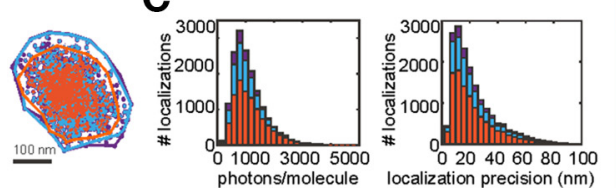

D

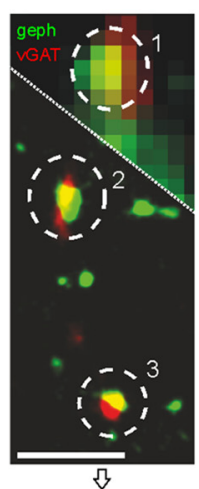

तु
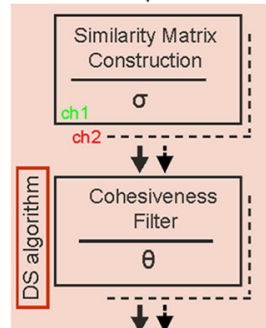

$\bar{\dagger} \bar{\dagger}$

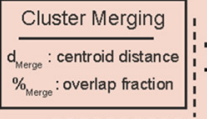
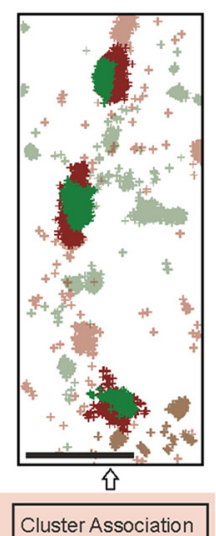
between channels $1 \mathrm{NN} \leq \mathrm{d}_{\mathrm{RG}}$
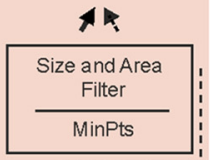

47

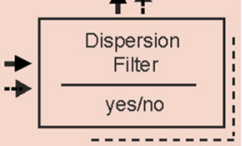

E 응
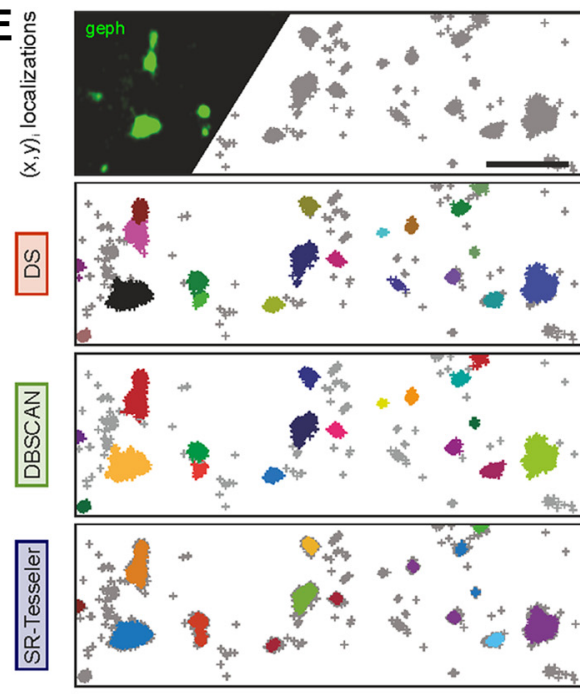

$F$
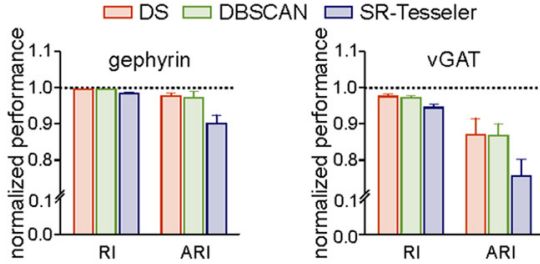

Figure 1. Single-molecule imaging and clustering analysis of gephyrin at the inhibitory synapse. $A$, Top, Representation of the recording protocol. After the beginning of photoactivation (black arrow), the intensity of the $405 \mathrm{~nm}$ laser was increased every 5000 frames while imaging with the $561 \mathrm{~nm}$ laser. Bottom, Histogram of the number of localizations obtained during the recording, grouped and color coded every 5000 frames. Note that the molecules detected before the photoactivation (gray) were not included in the analysis. $\boldsymbol{B}$, Top, Cluster radius (color code as in $\boldsymbol{A}$ ) at increasing intervals of frames after the photoactivation. Bottom, Representation of gephyrin cluster area (color code as in $A$ ) for the three frame intervals indicated above. $\boldsymbol{C}$, Corresponding histograms of the photons/molecule and localization precision for the three frame ranges considered. Color code as in $\boldsymbol{A}$. D, Implemented DS clustering algorithm. Top, Left, Representative input of the clustering algorithm; that is, a two-color SML image of gephyrin-Alexa Fluor647 and vGAT-Att0520 (the top portion of the image shows the corresponding wide field). Right, Representative output of the DS; that is, a cluster association between the two channels. Scale bar, $1 \mu \mathrm{m}$. Bottom, Flowchart of the clustering algorithm. Channel 1 (ch1, e.g., gephyrin) and channel 2 (ch2, e.g., VGAT) localizations follow independently the same pipeline: (1) definition of the similarity matrix ( $\sigma=60 \mathrm{~nm})$, (2) filter on cohesiveness $(\theta=0.95)$, (3) cluster merging according to $150 \mathrm{~nm}$ distance (approximately the maximal dimension of the expected clusters) and $0.001 \%$ overlap fraction, (4) possible dispersion filter (yes/no), (5) size filter $(>10$ locs) and area filter ( $<2$ nearest neighbors within a radius of $\sim 30 \mathrm{~nm}$ ), and (6) final association between clusters in the two channels located with a distance $<200 \mathrm{~nm}$. $\boldsymbol{E}$, Top, Representative SML image of gephyrin-Alexa Fluor647, shown in Gaussian (left) and scatter (right) rendering. Bottom panels show the output of DS, DBSCAN, and SR-Tesseler clustering algorithms applied to the image on top. Scale bar, $1 \mu \mathrm{m}$. F, Quantification of the Rand Index (RI) and the Adjusted Rand Index (ARI) to measure the clustering performace of the three different algoritms on gephyrin (left) and the noiser vGAT images (right) $(n=12)$.

$n$ refers to the number of individual synaptic cluster considered in the analysis.

\section{Results}

\section{Graph-based clustering algorithm for single-molecule} quantification of synaptic proteins

To quantify gephyrin clustering at the single-molecule level, we performed SML superresolution imagining of the inhibitory scaffold protein gephyrin tagged with the genetically encoded photoswitchable protein mEos3.2 expressed in hippocampal neurons. The advantage of mEos3.2 is that, despite its lower efficiency of photoactivation compared with other mEos variants (Durisic et al., 2012), it shows negligible oligomerization. To avoid overcounting or apparent clustering of molecules, the raw data of gephyrin-mEos3.2 emission were corrected for the intrinsic photoblinking of the converted mEos3.2 following the approach presented in Annibale et al. (2011) by introducing the empirical dark-state lifetime to group signals from the same blinking molecules (see Materials and Methods). To identify gephyrin clusters, SML was limited to the first 5000 frames after the photoactivation (Fig. 1A). Such temporal constraint minimizes the contribution of the autofluorescence that would overlay an additional background signal, potentially interfering with the computation of the molecules number and cluster area. Analyzing more frames or increasing the photoactivation laser intensity did not improve the identification of gephyrin molecules significantly, indicating that our experimental conditions allowed reliable single-molecule imaging of gephyrin in terms of cluster area, number of photons/molecule, and localization precision (Fig. $1 B, C)$.

To quantify gephyrin molecular density and cluster size, after performing SML, superresolution images were processed with a customized implemented version of a graph-based clustering algorithm (Pavan and Pelillo, 2007) called dominant set (DS). The main characteristics of the conventional DS clustering algorithm are that it relies on a limited number of parameters, it shows strong robustness to noise, and no global assumptions on the density of points per clusters drive the clusters selection. In the first step of our implemented version of the DS algorithm (Fig. $1 D$ ), all molecule localizations were mapped with a similarity matrix that quantifies the closeness of pairs of nodes. Importantly, this procedure strongly relies on the $\sigma$ parameters, which defines the minimum size of the identified clusters. Subsequently, the closeness of the points inside a cluster was assessed with the cohesiveness value $(\theta)$ that quantifies the average similarity among the elements of a cluster, thus allowing for signal identification and noise rejection (see Materials and Methods). A 
higher level of cohesiveness stands for highly structured clusters and, vice versa, low values indicate clusters with no significance or mainly composed of outliers. Furthermore, to handle the heterogeneous dimensions of the synaptic protein clusters, a cascade of postprocessing filters was implemented (Fig. $1 D$ ). In particular, we introduced a "merging" step that takes into account the closeness of the centroids and the convex hull $\psi_{\mathrm{i}}$ to circumvent oversegmentation, a dispersion filter to reject clusters with an anomalous spatial distribution, and a size filter to retain only clusters composed of a minimum number of localizations. Since the clustering process strictly depends on $\sigma$ with a direct impact on the spatial ranges of the identified clusters, the clustering performances of the DS algorithm have been tested for different $\sigma$ values. We experimentally tested different $\sigma$, ranging from $30-$ $220 \mathrm{~nm}$, and we identified the value $\sigma=60 \mathrm{~nm}$ as the most suitable to avoid oversegmentation and overassociation. In all of our analyses, the $\sigma$ value was kept constant to identify clusters with the same criteria and to achieve quantitative comparisons between samples. To confirm the appropriateness of the DS approach for synaptic proteins quantifications, we compared its clustering performances with those of other previously published algorithms (Fig. 1E). The same superresolution images were analyzed with DS method, with the better characterized densitybased method DBSCAN (Ester et al., 1996), and with the more recently proposed SR-Tesseler approach (Levet et al., 2015). The quantitative comparison of DS, DBSCAN, and SR-Tesseler was performed by measuring the matching quality between the cluster annotations and the predicted assignment of molecules-tocluster from the three methods. In particular, we estimated the goodness of the clustering by measuring two representative parameters, the Rand Index and the Adjusted Rand Index (Fig. 1F, left). We observed that the DS algorithm provided richer information on the estimated gephyrin clusters and showed comparable or better performances compared with DBSCAN and SR-Tesseler approaches. When the DS algorithm was used for the clustering of noisy SML images such as vGAT, it still outperformed the DBSCAN and the SR-Tesseler approach (Fig. 1F, right). Overall, our quantifications indicate that our implemented version of the DS algorithm avoids overassociation and oversegmentation of the data, representing a suitable computational instrument for quantitative analysis of superresolved clusters.

\section{Quantitative changes of superresolved synaptic and extrasynaptic gephyrin during GABAergic synaptic potentiation}

By processing the SML measurements with the aforementioned adapted DS-clustering algorithm, in a first set of analyses, we aimed at quantifying gephyrin molecular density and clusters size at synaptic and extrasynaptic compartments. Gephyrin-mEos3.2 was simultaneously imaged by PALM along with the presynaptic vGAT labeled with a specific Oyster650-conjugated antibody to visualize inhibitory synapses (Fig. 2A). Gephyrin clusters juxtaposed to vGAT puncta were defined as synaptic, whereas the remaining gephyrin clusters were considered extrasynaptic (Fig. $2 B)$. Although conventional microscopy detected only synaptic clusters, SML imaging revealed both synaptic ( $\sim 130 \mathrm{~nm}$ radius) and extrasynaptic ( $<100 \mathrm{~nm}$ radius) clusters. These latter were populated by significantly fewer gephyrin molecules than synaptic clusters [extrasyn: median $=142$, interquartile range $(\mathrm{IQR})=69-325$; syn: median $=702, \mathrm{IQR}=324-1165 ; p<$ 0.001 , Mann-Whitney test; Fig. 2C]. In addition, at extrasynaptic regions, gephyrin clusters exhibited significantly lower area and reduced density of molecules compared with synaptic compartments (Fig. 2C).

In a second set of experiments, we studied the gephyrin molecular clustering during chemically induced iLTP. This form of inhibitory synaptic plasticity has been reported previously to enhance of GABAergic synaptic currents by promoting the accumulation of GABAA receptors and gephyrin at postsynaptic sites (Petrini et al., 2014). By applying the methodology described above, we compared gephyrin aggregation in basal conditions and during iLTP (Fig. 2D). The expression of long-term synaptic plasticity induced substantial alterations of the gephyrin distribution at both extrasynaptic and synaptic sites. At synapses, a significant increase of gephyrin molecules per cluster was associated with unaffected cluster area, thus leading to increased density of gephyrin molecules per cluster (Fig. $2 E$ ). In contrast, at extrasynaptic sites, the same plasticity protocol induced a significant reduction of both the gephyrin molecule number per cluster and the gephyrin cluster area, leaving unchanged the density of gephyrin molecules per cluster (Fig. 2E). Chemical impairment of the iLTP-inducing protocol with $50 \mathrm{~mm}$ APV (see Materials and Methods) prevented gephyrin rearrangements and left synaptic and extrasynaptic gephyrin clusters unaffected (Fig. $2 F$ ). Furthermore, consistent with promoted gephyrin clustering during iLTP, we observed a significant increase in the number of synaptic clusters along the dendrites under plasticity conditions compared with control (Fig. 2G).

\section{Nanoscale arrangement of synaptic gephyrin clusters shifts toward nanodomains during iLTP}

Next, we exploited the SML approach (MacGillavry et al., 2013, Nair et al., 2013, Specht et al., 2013) to disclose specifically with nanometer accuracy the modification of the inner protein organization at the inhibitory synapse during iLTP. In basal conditions, synaptic gephyrin was mainly assembled in single-spot clusters, showing Gaussian-like density distribution (Fig. $3 A$ ). However, in a subset of synapses, the map of the gephyrin local density revealed a multispot nanodomain organization (Fig. 3B). After the induction of inhibitory LTP, the fraction of the multispot synapses increased significantly (from $27 \pm 15 \%$ in the basal condition to $49 \pm 10 \%$ with iLTP, $n=80$ and 107 , respectively, $p<0.01$, Student's $t$ test; Fig. $3 C$ ) at the expense of the single-spot ones (basal: $73 \pm 15 \%$; iLTP: $51 \pm 10 \%, n=170$ and 105, respectively, $p<0.01$, Student's $t$ test; Fig. $3 C$ ). Moreover, in iLTP conditions, gephyrin potentiation, the increased number of molecules per cluster, cluster area, and density of molecules per clusters, was observed selectively at multispot synapses, whereas single-spot synapses were similar both in basal and in plasticity conditions (Fig. 3D). These data indicate that, after the induction of iLTP, the increased clustering of synaptic gephyrin is accompanied by the formation of gephyrin nanodomains and that only a subset of inhibitory synapses undergoes plastic changes. Remarkably, monospot and multispot clusters were also detected by SML analysis of endogenous gephyrin in STORM experiments in nontransfected neurons (Fig. $3 E$ ), ruling out the possibility that gephyrin nanodomains could arise from gephyrin-mEos3.2 overexpression. The same STORM approach revealed that the effect of the iLTP-inducing protocol on endogenous gephyrin was comparable to that reported on gephyrin-mEos3.2. Indeed, we observed a significantly larger density of endogenous gephyrin synaptic clusters along dendrites than in controls (Fig. $3 F$ ) and a higher fraction of multispot synapses compared with control conditions (ctrl: $28 \pm 2 \%$; iLTP: $53 \pm 6 \% ; n=203$ and 176, respectively, $p<0.05$, Student's $t$ test; Fig. $3 G$ ), with the concom- 


\section{A}
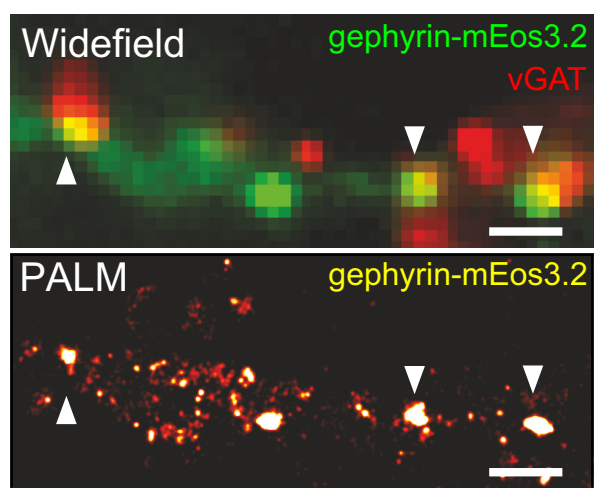

C

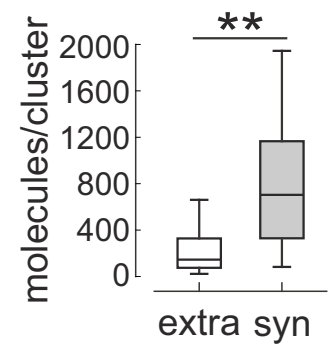

D
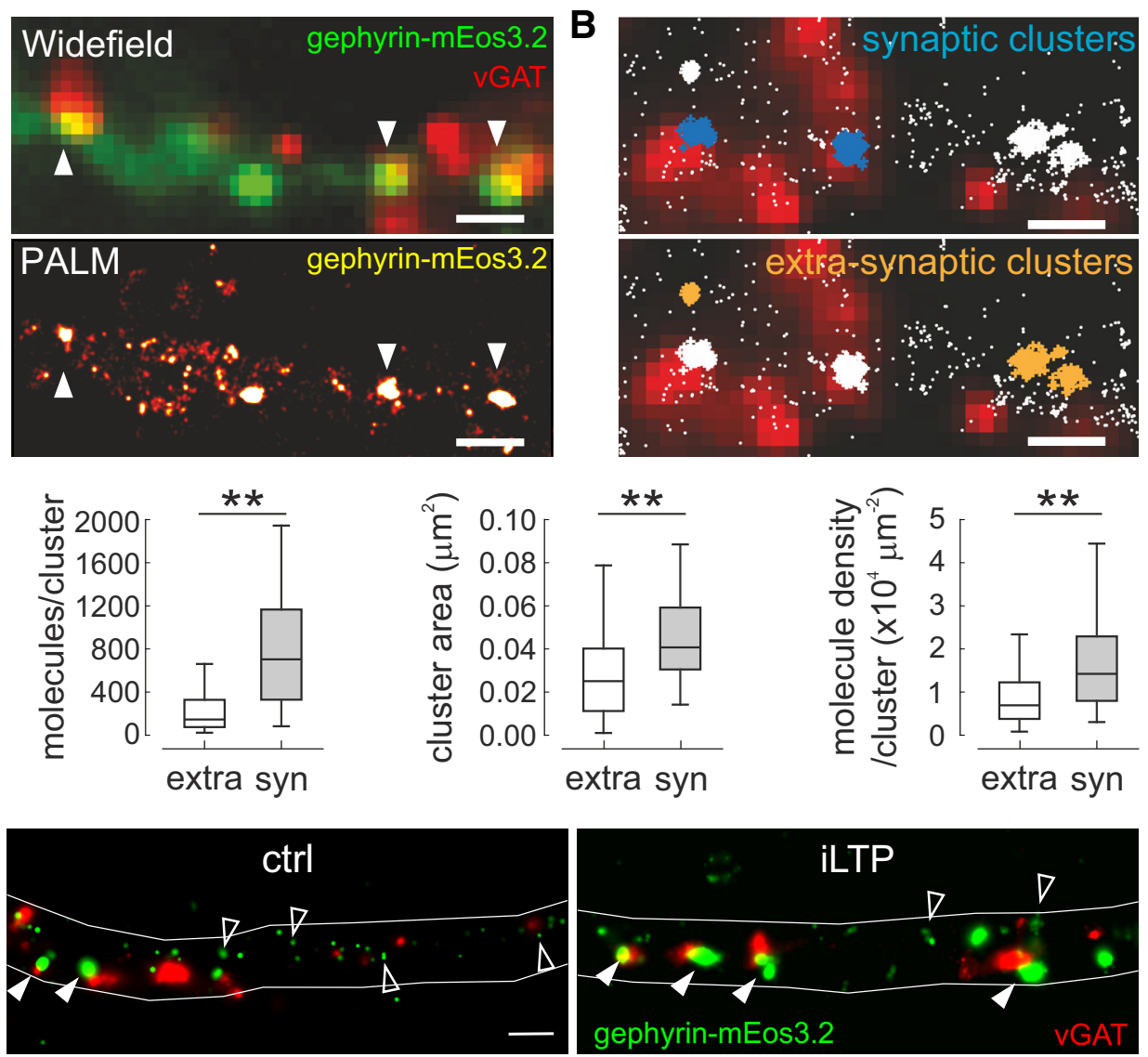

E
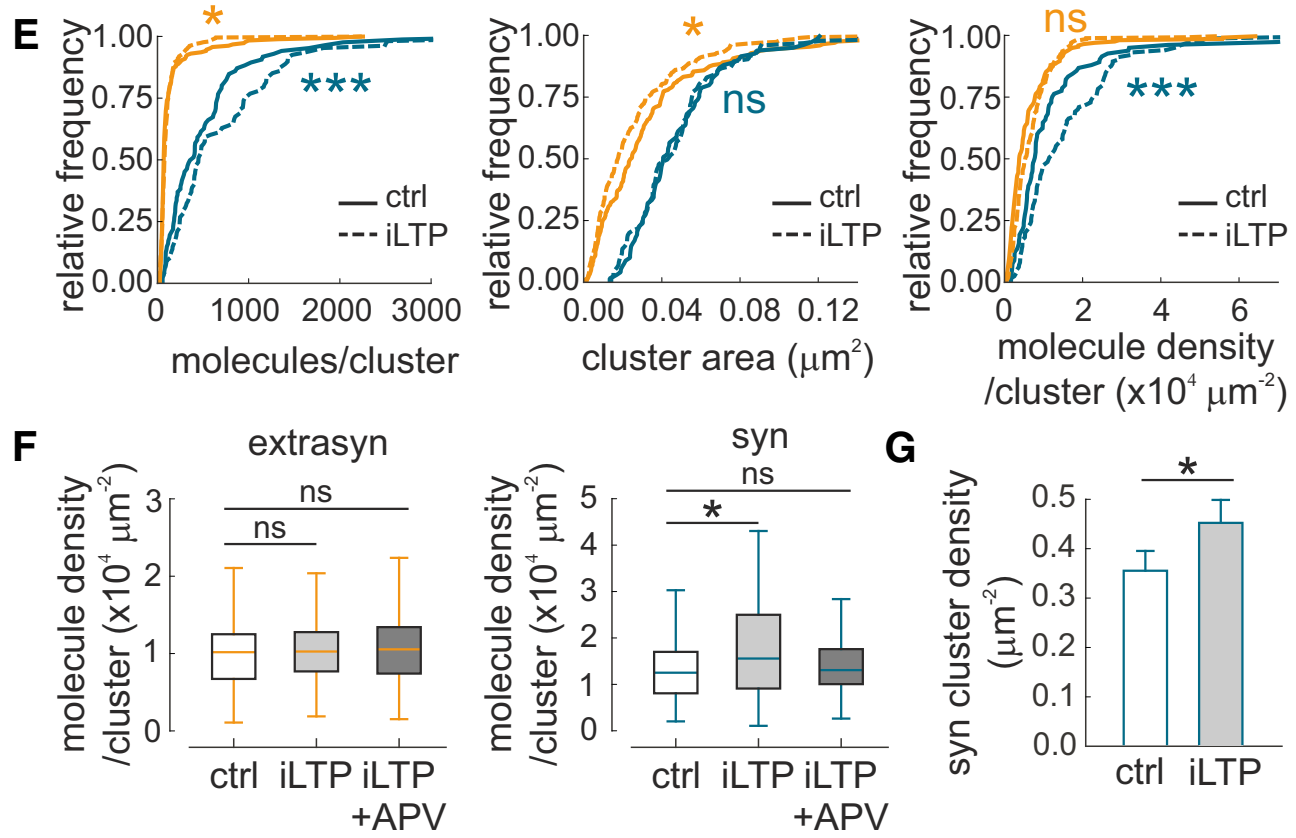

G

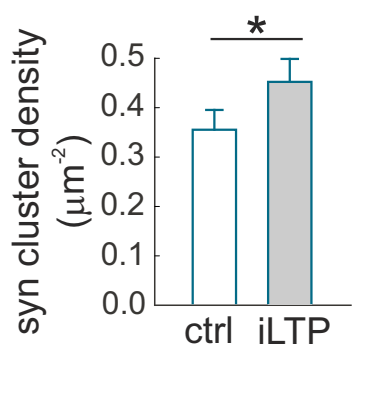

Figure 2. Redistribution of extrasynaptic gephyrin to synaptic compartments during iLTP. $A$, Top, Wide-field image of gephyrin-mEos3.2 (green) and vGAT (red) along a neuronal dendrite. Note gephyrin clusters juxtaposed to presynaptic vGAT (arrowheads). Bottom, PALM reconstruction of gephyrin-mEos3.2 shown above. Scale bars, $1 \mu \mathrm{m}$. $\boldsymbol{B}$, Representative SML images of gephyrin (white) superimposed upon the wide-field image of the presynaptic vGAT (red). Top, Synaptic clusters are represented in blue. Bottom, Extrasynaptic clusters are shown in orange. Scale bars, $1 \mu \mathrm{m}$. C, Quantification of gephyrin number of molecules/cluster, area, and molecule density/cluster quantified at extrasynaptic and synaptic compartments $(n=221$ and 85 , respectively), MannWhitney test. D, Representative dual color SML images of gephyrin (green) and vGAT (red) along neuronal dendrites (solid lines) in control (left) and iLTP (right). Extrasynaptic (empty arrowheads) and synaptic gephyrin juxtaposed to vGAT (plain arrowheads) are shown. Scale bar, $0.5 \mu \mathrm{m}$. $\boldsymbol{E}$, Cumulative distribution of gephyrin number of molecules/cluster (left), cluster area (middle), and molecule density/cluster (right) at extrasynaptic (orange, $n=185-260$ ) and synaptic (blue, 85-107) compartments before (ctr) and during iLTP. Kolmogorov-Smirnov test. $\boldsymbol{F}$, APV prevents the gephyrin increase elicited by the iLTP-inducing protocol at extrasynaptic (left, $n=250-386-611$, for ctrl, iLTP and iLTP + APV, respectively) and synaptic (right, $n=87-146-157$, for ctrl, iLTP and iLTP + APV, respectively) compartments. One-way ANOVA (Kruskal-Wallis test), followed by Dunn's post test. G, Number of synaptic gephyrin clusters per dendrite area under basal conditions (ctrl) and during synaptic plasticity (iLTP). $n=17$ and 23 dendrites in ctrl and iLTP, respectively. Student's $t$ test, n.s. nonsignificant, ${ }^{*} p<0.05,{ }^{* *} p<0.01,{ }^{* * *} p<0.001$. 
A

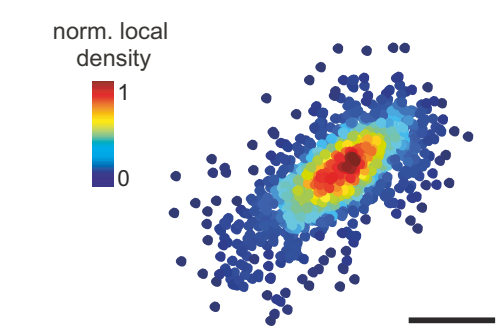

D

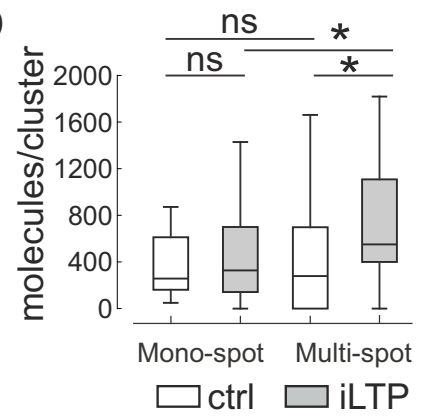

B
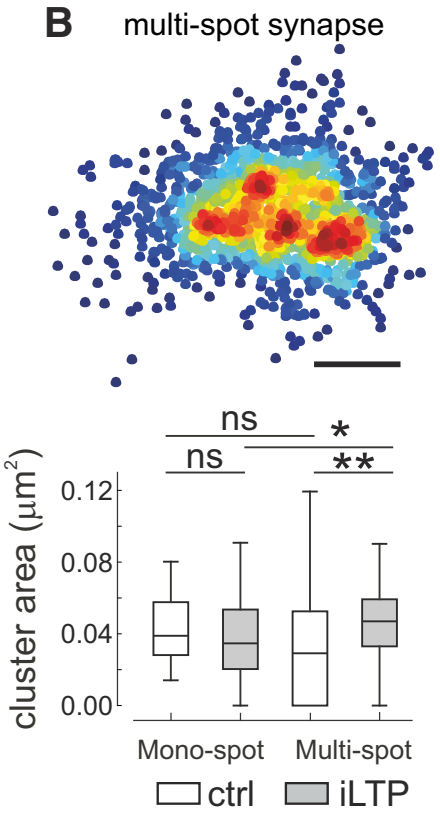

C
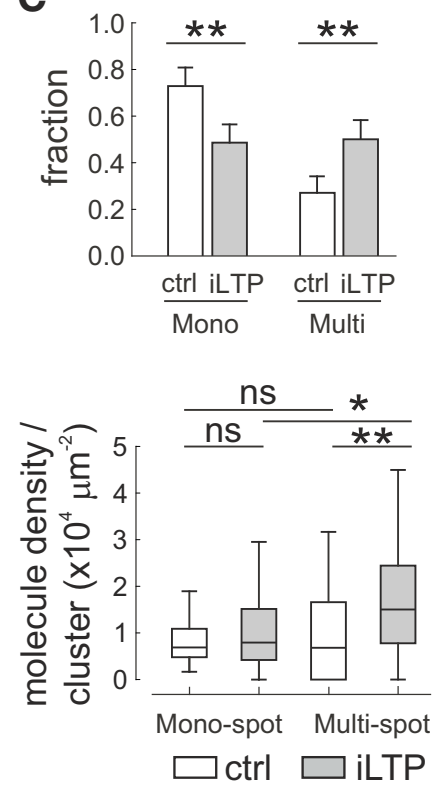

E

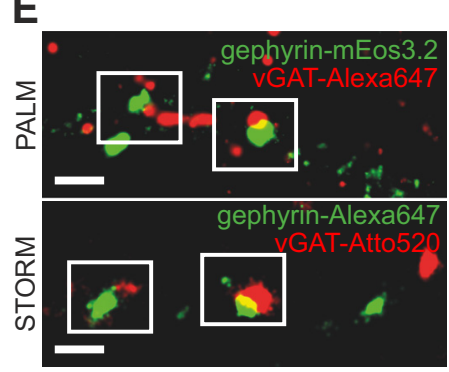

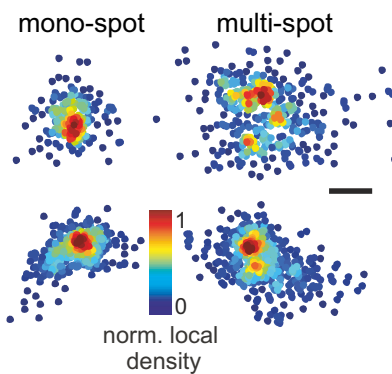

$F$

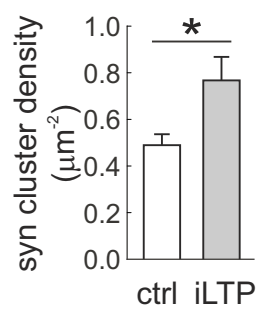

G

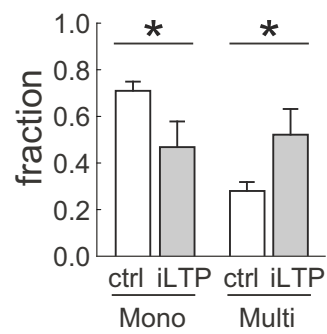

Figure 3. Increased multispot nanoscale arrangement of synaptic gephyrin clusters during iLTP. A, B, Pseudocolor representation of SML gephyrin-mEos3.2 normalized local density (radius $\sim 15$ $\mathrm{nm}$ ) reveals the nanoscale organization of synaptic gephyrin in monospot $(\boldsymbol{A})$ and multispot $(\boldsymbol{B})$ clusters. Scale bars, $100 \mathrm{~nm}$. $\boldsymbol{C}$, Fractions of synaptic clusters organized in monospot or multispot configurations in basal conditions (white bars, $n=120,51$, respectively) and iLTP (gray bars, $n=53,52$, respectively). Data are presented as mean \pm SD (D). Molecular characterization [number of molecules (left), area (middle), and density (right)] of monospot and multispot gephyrin synaptic clusters are shown in control (white bars) and during synaptic plasticity (gray bars). Data are presented as median and interquartile range. One-way ANOVA (Kruskal-Wallis test). $\boldsymbol{E}$, Endogenous and overexpressed gephyrin nanodomains. Left, Representative dual color SML images of gephyrin-mEos3.2 (top, PALM) and endogenous gephyrin (bottom, STORM), imaged along with vGAT-Alexa Fluor 647 and vGAT-Atto520, respectively. Scale bar, $0.5 \mu$ m. Right, Magnified normalized local density plots of the synapses framed on the left show the monospot and multispot organization of both gephyrin-mEos 3.2 and endogenous gephyrin. Scale bar, $100 \mathrm{~nm}$. $F$, Number of synaptic endogenous gephyrin clusters per dendrite area under basal conditions (ctrl) and during synaptic plasticity (iLTP) quantified in STORM. $n=12$ and 13 in ctrl and iLTP, respectively. $G$, Fractions of synaptic clusters organized in monospot and multispot configuration in basal conditions (white bars, $n=147$ and 56, respectively) and iLTP (gray bars, $n=81$ and 94 , respectively) quantified in STORM. Data are presented as mean \pm SD, n.s. nonsignificant, ${ }^{*} p<0.05,{ }^{* *} p<0.01$.

itant reduction of single-spot ones (ctrl: $72 \pm 2 \%$; iLTP: $47 \pm 6 \%$; $n=203$ and 176, respectively, $p<0.05$, Student's $t$ test; Fig. $3 G$ ). These data indicate a similar reorganization of recombinant and endogenous gephyrin during iLTP (cf. Fig. 3C,G).

\section{Functional role of gephyrin synaptic nanodomains}

Although synaptic nanodomains have been described previously (MacGillavry et al., 2013, Nair et al., 2013, Specht et al., 2013), their functional role in synaptic plasticity is poorly understood. To address this issue, we exploited computer modeling (see Materials and Methods) to simulate the activation of individual synaptic GABAA receptors by the release of a single synaptic vesicle in a realistic synaptic environment (Petrini et al., 2011). In particular, the GABA release in the synaptic cleft was simulated by using the diffusion equation with a 3D free boundary condition and a diffusion coefficient corrected by the tortuosity (Petrini et al., 2011), whereas the gating of synaptic GABAA receptors was simulated by using the Jones and Westbrook model (Jones and Westbrook, 1995), a kinetic scheme that satisfactorily reproduces the time course of IPSCs elicited by synaptic-like brief agonist exposures (Barberis et al., 2011). By assuming the matching between receptor density and gephyrin distribution (Specht et al., 2013), we considered different spatial organizations of GABAA receptors on the postsynaptic disk, including the following: (1) Gaussian distribution (monospot synapse), (2) eight or (3) four randomly dispersed nanodomains, and (4) four fixed nanodomains showing equal distance $(0.1 \mu \mathrm{m})$ from the disk center (Fig. $4 A$ ). These receptor distributions were associated with a silhouette coefficient, a parameter that defines the degree of clustering (see Materials and Methods; Fig. 4A). Receptors were activated by simulated GABA released from sites randomly distributed in the disk area (see Materials and Methods). We found that, in the multispot configuration, the CV of simulated IPSC peak amplitude was lower with respect to that of simulated IPSCs elicited at monospot Gaussian synapses (Fig. 4B, C). Moreover, in the random multispot configurations (four and eight nanodomains), the CV decreased monotonically with increasing silhouette values (i.e., clustering; Fig. 4D). Similar correlations between $\mathrm{CV}$ 

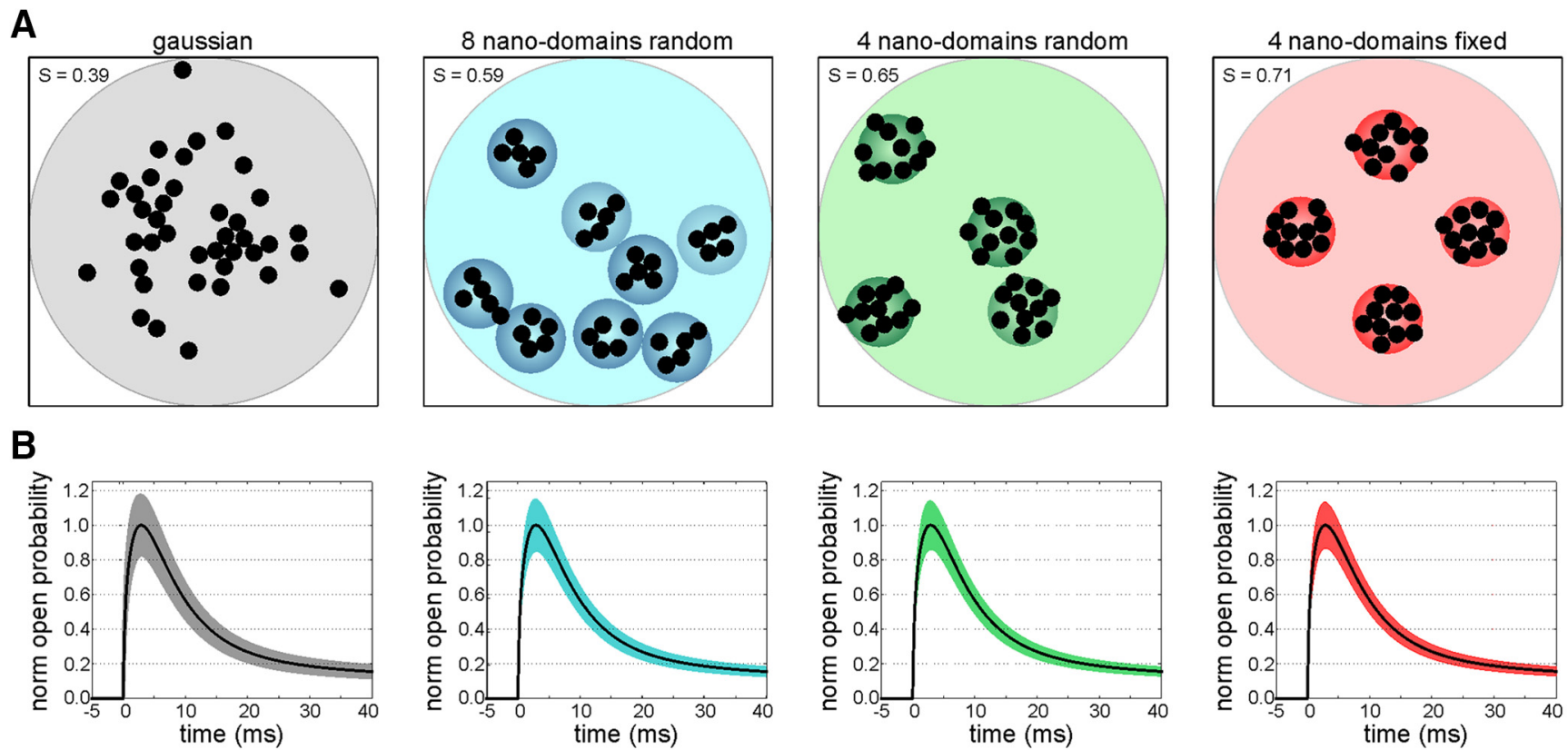

\section{C}

\section{D}

\section{$E$}

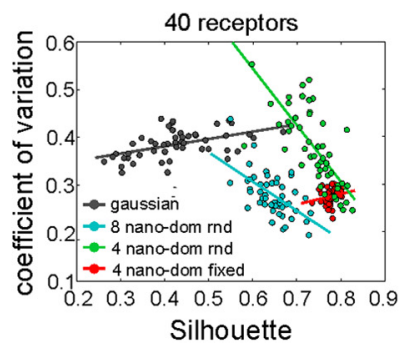

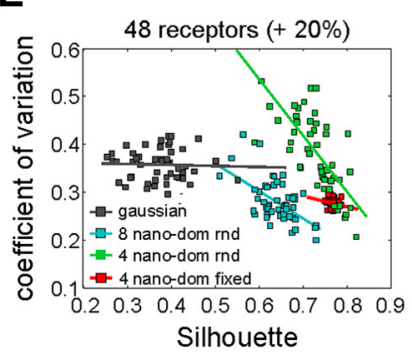

$\mathbf{F}$

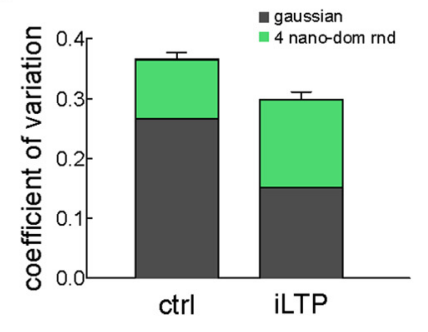

Figure 4. Model simulations of synaptic receptor activated in different spatial distributions. $A$, Example of GABAA receptors distributions in the synaptic disk ranked from left to right according to increasing silhouette coefficients (S). Please note that each of the configurations shown represents one realization of the 50 considered for the calculation of the IPSC CV. $\boldsymbol{B}$, Normalized mean open probabilities of receptors (black curves, representing IPSC time course) in the spatial configurations shown in $A$. Responses are elicited by the simulated release of GABA activating GABAA receptors in the synaptic disk. Colored areas represent the CV of the open probability time course in the different configurations. C, Mean CV at the peak of simulated IPSCs in the different configurations. Please note that the IPSC variability in the nanodomain configurations is lower than that obtained in the Gaussian configuration. D, Dependence of the IPSCs CV on the silhouette coefficient in a $0.3 \mu \mathrm{m}$ synaptic disk radius containing 40 receptors. Each dataset shown in colored circles represents the 50 realizations analyzed and the associated regression line. Note that in both the 4 and 8 nanodomain random configurations (green and blue circles, respectively) the CV of the peak open probability decreases at increasing values of the silhouette coefficient, as indicated by a clear negative regression slope value (slope of regression line with $95 \%$ confidence interval $=-1.18 \pm 0.29$ and $-0.61 \pm 0.12$ for the 4 and 8 nanodomains, respectively). The four fixed nanodomain configuration (red circles) and the Gaussian configuration (gray circles), in contrast, showed no negative CV-silhouette relation. Please note that, being that the variability of the four fixed nanodomain configuration merely due to the different distribution of the receptors within each of the four fixed nanodomains, the points of the distribution are much less dispersed whit respect to those of all the other configurations. $\boldsymbol{E}$, Same data as in D in a $0.3 \mu \mathrm{m}$ synaptic disk radius enriched with $+20 \%$ receptors (i.e., 48 receptors), a condition observed during iLTP (Petrini et al., 2014 ). Note that the CV reduction as a function of the silhouette persists in the random 4 and 8 nanodomains configurations when the $20 \%$ increase of receptor is simulated (slope of regression lines with $95 \%$ confidence intervals $=-1.18 \pm 0.30$ and $-0.57 \pm 0.24$ for the 4 and 8 nanodomains, respectively), whereas no significant negative correlation was observed for the fixed 4 nanodomains and the Gaussian configurations. $\boldsymbol{F}$, Impact of synapse nanofragmentation during iLTP on IPSC variability. Average CV of simulated IPSCs weighted for the different synapse configurations; that is, monospot (gray) and multispot (4 random nanodomains, green) in control conditions (mono: $73 \pm 15 \%$ and multi: $27 \pm 15 \%$ ) and during iLTP (mono: $51 \pm 10 \%$ and multi: $49 \pm 10 \%$ ) as in Figure 3C. In the iLTP condition, the number of synaptic receptors was increased by $20 \%$ (ctrl: 40 receptors; iLTP: 48 receptors).

and silhouette were obtained at different synaptic disk radius and when the number of receptors was increased by $20 \%$ (Fig. $4 E$ ), a condition observed experimentally during iLTP (Petrini et al., 2014). Importantly, the silhouette coefficients of simulated multispot synapses was consistent with that of experimental multispot synapses under the iLTP conditions $(S=0.82 \pm 0.02, n=$ 15). To investigate the impact of the synaptic nanofragmentation on IPSC variability during synaptic plasticity, we evaluated the simulated $\mathrm{CV}$, taking into account the experimentally observed increase in multispot synapses in iLTP conditions. According to the values reported in Figure $3 C$, the CV in iLTP was calculated as the weighted average of the CV obtained with 48 receptors in $51 \pm 10 \%$ single-spot synapses (Gaussian) and in $49 \pm 10 \%$ multispot synapses (with four to eight nanodomains random;
Fig. 4F). Analogously, we calculated the control $\mathrm{CV}$ as the weighted average of the CV obtained with 40 receptors in $73 \pm$ $15 \%$ single-spot synapses (Gaussian) and in $27 \pm 15 \%$ multispot synapses (with four to eight nanodomains random). Our model simulations indicate an $\sim 18 \%$ reduction of the synaptic current CV during iLTP. Next, we recorded sIPSCs before and after the induction of iLTP in cultured hippocampal neurons to evaluate changes in experimental CV. After the induction of iLTP, the sIPSC peak CV was reduced significantly $(\sim 22 \%)\left(\mathrm{CV}_{\text {before }}=\right.$ $0.64 \pm 011 \%, \mathrm{CV}_{\mathrm{after}}=0.50 \pm 0.06 \%, n=15, p<0.05$ paired $t$ test). Interestingly, these results were consistent with the weighted average CV of simulated currents. Overall, our data reveal that the fragmentation of receptor postsynaptic cluster stabilizes the amplitude of IPSCs by reducing current vari- 
ability, thus representing a novel determinant for synaptic plasticity.

\section{Discussion}

In the present work, SML techniques, graph-based data-clustering analysis, and model simulations allowed us to characterize the spatial reorganization of gephyrin during inhibitory synaptic plasticity. Previous studies have identified the insertion of GABAA receptors at postsynaptic sites as a determinant for the potentiation of GABAergic responses (Nusser et al., 1998, Petrini et al., 2014). In addition, with an electron microscopy approach, plastic changes of the inhibitory synapse have been associated with modifications of the postsynaptic density (PSD) structure (Lushnikova et al., 2011). We report here that expression of iLTP increases the fraction of synapses exhibiting gephyrin nanodomains and we provide a functional role for such molecular rearrangement in the potentiation of inhibitory synaptic responses. Indeed, our model simulations and experimental evidence demonstrate that gephyrin subsynaptic fragmentation minimizes the inhibitory current fluctuations. This indicates that iLTP expression involves, not only the increase of maximal synaptic response, but also the stabilization of the synaptic current amplitude. However, at this stage, we cannot rule out that presynaptic mechanisms may also contribute to the reduction of IPSC variability observed experimentally. Indeed, it has been shown that activation of NMDA receptors at GABAergic terminals may increase the amplitude of synaptic currents by increasing GABA release (Duguid and Smart, 2004). Although this presynaptic potentiation has been shown to last only few minutes after NMDA application, it cannot be excluded that residual presynaptic effects may persist at times in which we evaluate the postsynaptic iLTP, thus partially contaminating the postsynaptic source of sIPSC CV. Although subsynaptic nanodomains have been reported at both glutamatergic and GABAergic synapses (MacGillavry et al., 2013, Nair et al., 2013, Specht et al., 2013), the molecular mechanisms that promote their formation remain obscure. It has been proposed previously that the formation of receptor-scaffold clusters of size comparable to those found at central synapses is achieved by the equilibrium between aggregating forces (scaffold-scaffold, receptor-scaffold) and repulsive forces due to the steric hindrance of diffusing neurotransmitter receptors (Haselwandter et al., 2011, Haselwandter et al., 2015). It can be hypothesized that similar passive mechanisms would also take place in the establishment of subsynaptic nanodomains in response to plasticityinduced alterations of receptors diffusion and/or changes of scaffold-scaffold and receptor-scaffold interactions. In addition, as discussed in Nair et al. (2013), nanodomains could be orchestrated to match presynaptic determinants through the transsynaptic NLG-Neurexin complexes. In the present study, we assumed that GABAA receptors show nanodomain distribution comparable to gephyrin. This might be considered a fair assumption based on previous superresolution investigations at excitatory and inhibitory synapses reporting that AMPA and glycine receptors are organized in nanodomains (MacGillavry et al., 2013; Nair et al., 2013; Specht et al., 2013). The SML results shown here support the hypothesis that, during the expression of iLTP, extrasynaptic gephyrin clusters are depleted and extrasynaptic gephyrin molecules are displaced to become incorporated into synaptic clusters. Our approach revealed that such gephyrin synaptic recruitment involves the reduction of the area (but not the density) of extrasynaptic gephyrin clusters and the increase of the density (but not the area) of synaptic gephyrin clusters. Previous studies exploiting the immunogold labeling technique combined with electron microscopy, however, reported increased synaptic size area after inhibitory potentiation induced by a variety of plasticity protocols including a temporal-lobe epilepsy model (Nusser et al., 1998), glucose-oxygen deprivation (Lushnikova et al., 2011), and theta burst stimulations (Bourne and Harris, 2011). This discrepancy can be explained by considering that the aforementioned studies found significant plasticity-related PSD modifications at times $>1 \mathrm{~h}$, whereas in the present work, due to experimental limitations, we examined gephyrin distribution in the first $30 \mathrm{~min}$ after the delivery of the plasticity induction protocol. In support of the hypothesis that the molecular events responsible for inhibitory potentiation may vary at different time points after the plasticity induction, we previously identified $45 \mathrm{~min}$ as a cutoff time for increased gephyrin protein synthesis to start contributing to iLTP expression (Petrini et al., 2014). Therefore, it is reasonable to hypothesize that the remodeling of inhibitory postsynaptic density could significantly differ in "early" or "late" phases of synaptic plasticity, the first mainly depending on protein redistribution and the latter sustained by de novo protein synthesis and characterized by a more substantial protein rearrangement. Furthermore, a timerelated dependence of inhibitory plasticity process are supported by several studies showing that, at late stages, iLTP occurs in concomitance with glutamatergic LTP, indicating a homeostatic regulation of excitation and inhibition (Bourne and Harris, 2011, Lushnikova et al., 2011, Flores et al., 2015), whereas early iLTP is associated with depression of excitatory synapses (Marsden et al., 2010, Petrini et al., 2014). This scenario suggests a sequence of different dendritic molecular remodeling after the onset of iLTP, coordinated at both GABAergic and glutamatergic synapses. Overall, our findings identify the nanoscale organization of inhibitory synaptic proteins as an important determinant for inhibitory synaptic plasticity, thus adding a further level of complexity to the regulation of the neuronal network activity by plastic inhibitory synaptic signals.

\section{References}

Annibale P, Vanni S, Scarselli M, Rothlisberger U, Radenovic A (2011) Quantitative photo activated localization microscopy: unraveling the effects of photoblinking. PLoS One 6:e22678. CrossRef Medline

Barberis A, Petrini EM, Mozrzymas JW (2011) Impact of synaptic neurotransmitter concentration time course on the kinetics and pharmacological modulation of inhibitory synaptic currents. Front Cell Neurosci 5:6. CrossRef Medline

Bourne JN, Harris KM (2011) Coordination of size and number of excitatory and inhibitory synapses results in a balanced structural plasticity along mature hippocampal CA1 dendrites during LTP. Hippocampus 21:354-373. CrossRef Medline

Dani A, Huang B, Bergan J, Dulac C, Zhuang X (2010) Superresolution imaging of chemical synapses in the brain. Neuron 68:843-856. CrossRef Medline

Deschout H, Cella Zanacchi F, Mlodzianoski M, Diaspro A, Bewersdorf J, Hess ST, Braeckmans K (2014a) Precisely and accurately localizing single emitters in fluorescence microscopy. Nat Methods 11:253-266. CrossRef Medline

Deschout H, Shivanandan A, Annibale P, Scarselli M, Radenovic A (2014b) Progress in quantitative single-molecule localization microscopy. Histochem Cell Biol 142:5-17. CrossRef Medline

Duguid IC, Smart TG (2004) Retrograde activation of presynaptic NMDA receptors enhances GABA release at cerebellar interneuron-Purkinje cell synapses. Nat Neurosci 7:525-533. CrossRef Medline

Durisic N, Godin AG, Wever CM, Heyes CD, Lakadamyali M, Dent JA (2012) Stoichiometry of the human glycine receptor revealed by direct subunit counting. J Neurosci 32:12915-12920. CrossRef Medline

Ester M, Kriegel HP, Sander J, Xu X (1996) A density based algorithm for discovering clusters in large spatial database with noise. Proceedings of 
the 2nd International Conference on Knowledge Discovery and Data Mining 34:226-231.

Fernández-Suárez M, Ting AY (2008) Fluorescent probes for super-resolution imaging in living cells. Nat Rev Mol Cell Biol 9:929-943. CrossRef Medline

Flores CE, Nikonenko I, Mendez P, Fritschy JM, Tyagarajan SK, Muller D (2015) Activity-dependent inhibitory synapse remodeling through gephyrin phosphorylation. Proc Natl Acad Sci U S A 112:E65-E72. CrossRef Medline

Haselwandter CA, Calamai M, Kardar M, Triller A, da Silveira RA (2011) Formation and stability of synaptic receptor domains. Phys Rev Lett 106: 238104. CrossRef Medline

Haselwandter CA, Kardar M, Triller A, da Silveira RA (2015) Self-assembly and plasticity of synaptic domains through a reaction-diffusion mechanism. Phys Rev E Stat Nonlin Soft Matter Phys 92:032705. CrossRef Medline

Jones MV, Westbrook GL (1995) Desensitized states prolong GABAA channel responses to brief agonist pulses. Neuron 15:181-191. CrossRef Medline

Levet F, Hosy E, Kechkar A, Butler C, Beghin A, Choquet D, Sibarita JB (2015) SR-Tesseler: a method to segment and quantify localizationbased super-resolution microscopy data. Nat Methods 12:1065-1071. CrossRef Medline

Lushnikova I, Skibo G, Muller D, Nikonenko I (2011) Excitatory synaptic activity is associated with a rapid structural plasticity of inhibitory synapses on hippocampal CA1 pyramidal cells. Neuropharmacology 60:757764. CrossRef Medline

MacGillavry HD, Song Y, Raghavachari S, Blanpied TA (2013) Nanoscale scaffolding domains within the postsynaptic density concentrate synaptic AMPA receptors. Neuron 78:615-622. CrossRef Medline

Marsden KC, Shemesh A, Bayer KU, Carroll RC (2010) Selective translocation of $\mathrm{Ca} 2+/$ calmodulin protein kinase IIalpha (CaMKIIalpha) to inhibitory synapses. Proc Natl Acad Sci U S A 107:20559-20564. CrossRef Medline
Nair D, Hosy E, Petersen JD, Constals A, Giannone G, Choquet D, Sibarita JB (2013) Super-resolution imaging reveals that AMPA receptors inside synapses are dynamically organized in nanodomains regulated by PSD95. J Neurosci 33:13204-13224. CrossRef Medline

Nusser Z, Hájos N, Somogyi P, Mody I (1998) Increased number of synaptic GABA(A) receptors underlies potentiation at hippocampal inhibitory synapses. Nature 395:172-177. CrossRef Medline

Pavan M, Pelillo M (2007) Dominant sets and pairwise clustering. IEEE Transactions on Pattern Analysis and Machine Intelligence 29:167-172. CrossRef Medline

Petrini EM, Nieus T, Ravasenga T, Succol F, Guazzi S, Benfenati F, Barberis A (2011) Influence of GABAAR monoliganded states on GABAergic responses. J Neurosci 31:1752-1761. CrossRef Medline

Petrini EM, Ravasenga T, Hausrat TJ, Iurilli G, Olcese U, Racine V, Sibarita JB, Jacob TC, Moss SJ, Benfenati F, Medini P, Kneussel M, Barberis A (2014) Synaptic recruitment of gephyrin regulates surface GABAA receptor dynamics for the expression of inhibitory LTP. Nat Commun 5:3921. CrossRef Medline

Sigrist SJ, Sabatini BL (2012) Optical super-resolution microscopy in neurobiology. Curr Opin Neurobiol 22:86-93. CrossRef Medline

Specht CG, Izeddin I, Rodriguez PC, El Beheiry M, Rostaing P, Darzacq X, Dahan M, Triller A (2013) Quantitative nanoscopy of inhibitory synapses: counting gephyrin molecules and receptor binding sites. Neuron 79:308-321. CrossRef Medline

Szymborska A, de Marco A, Daigle N, Cordes VC, Briggs JA, Ellenberg J (2013) Nuclear pore scaffold structure analyzed by super-resolution microscopy and particle averaging. Science 341:655-658. CrossRef Medline

Ulbrich MH, Isacoff EY (2007) Subunit counting in membrane-bound proteins. Nat Methods 4:319-321. Medline

Xu K, Zhong G, Zhuang X (2013) Actin, spectrin, and associated proteins form a periodic cytoskeletal structure in axons. Science 339:452-456. CrossRef Medline 